\title{
MUGSHOT IDENTIFICATION FROM MANIPULATED FACIAL IMAGES
}

\section{CHENNAMMA H.R. ${ }^{1}$ AND LALITHA RANGARAJAN²}

${ }^{1}$ Department of MCA, Sri Jayachamarajendra College of Engineering, Mysore, India.

2Dept. Of Studies in Computer Science, University of Mysore, Mysore, India.

*Corresponding Author: Email- 1anusha_hr@rediffmail.com and 2lali85arun@yahoo.co.in

Received: April 12, 2012; Accepted: May 15, 2012

\begin{abstract}
Editing digital images is ubiquitous. Identification of deliberately modified facial images is a new challenge for face identification system. In this paper, we address the problem of identification of a face or person from heavily altered facial images. Query is a manipulated or transformed face image and the system reports back the determined identity from a database of known individuals. Such a system can be useful in mugshot identification in which mugshot database contains two views (frontal and profile) of each criminal. We considered only frontal view from the available database for face identification. We propose SIFT features for efficient face identification in this scenario. Further comparative analysis has been given with well known eigenface approach. Experiments have been conducted with real case images to evaluate the performance of both methods.
\end{abstract}

Keywords- Mugshots, Image Tampering, Face Identification, SIFT, PCA.

Citation: Chennamma H.R. and Lalitha Rangarajan (2012) Mugshot Identification from Manipulated Facial Images. International Journal of Machine Intelligence, ISSN: 0975-2927 \& E-ISSN: 0975-9166, Volume 4, Issue 1, pp.-407.

Copyright: Copyright@2012 Chennamma H.R. and Lalitha Rangarajan. This is an open-access article distributed under the terms of the Creative Commons Attribution License, which permits unrestricted use, distribution, and reproduction in any medium, provided the original author and source are credited. 